\title{
Skeletal Diseases in Cushing's Syndrome: Osteoporosis versus Arthropathy
}

\author{
Gregory Kaltsas Polyzois Makras \\ Department of Pathophysiology, National University of Athens, Athens, Greece
}

\section{Key Words}

Osteoporosis · Cushing's syndrome • Avascular necrosis •

Bisphosphonates $\cdot$ Cortisol $\cdot$ Bone mineral density

\begin{abstract}
Structural and functional impairment of the skeletal system remains an important cause of morbidity and disability in patients with Cushing's syndrome (CS). Glucocorticoid (GC) excess inhibits bone formation and calcium absorption from the gut, increases bone resorption, and alters the secretion of gonadotropin and growth hormones, cytokines and growth factors influencing bone. Both overt and subtle endogenous hypercortisolism affect bone, leading to vertebral fractures in up to $70 \%$ of patients. Fracture risk is related to age at onset, duration and severity of the disease and individual susceptibility to GCs that is genetically determined. Bone mineral density (BMD) measurement at the lumbar spine should be performed as a screening test in all patients with CS due to the preferential loss of trabecular bone induced by GCs. The higher risk of fractures at comparable $B M D$ values with controls suggests that bone quality features, not assessed by routine BMD approaches, are also important and should be addressed when indicated applying specific radiological means. Successful treatment of GC excess is associated with improvement in bone mass which, although delayed and often incomplete, reduces the risk of
\end{abstract}

osteoporotic fractures. Bisphosphonates can induce a more rapid improvement in BMD than cortisol normalization alone and can be used in patients with increased risks for further fractures and/or persistent hypercortisolemia to prevent further bone loss. Anabolic agents have not as yet been systemically used. Avascular necrosis, mainly of the femoral neck, and growth arrest in children are the most common skeletal disorders unrelated to osteoporosis encountered in patients with endogenous hypercortisolism.

Copyright $\odot 2010$ S. Karger AG, Basel

\section{Introduction}

Glucocorticoids (GCs) exert multiple effects on bone and GC-induced osteoporosis is the commonest cause of secondary osteoporosis. However, the effects that GCs exert on bone metabolism are hampered by the specific effects of the underlying disease that GCs are prescribed for. Recently, a substantial amount of data has been gathered from studies looking at the effects that GC excess exerts on bone denoted of any other confounding factors such as in cases of endogenous hypercortisolism (Cushing's syndrome; CS). This paper addresses the pathogenesis, incidence, prediction and treatment of osteoporosis in patients with CS and discusses other less common, related arthropathies encountered in such patients.

\section{KARGER}

Fax +4161306 1234

E-Mail karger@karger.ch

www.karger.com
(C) 2010 S. Karger AG, Basel

0028-3835/10/0925-0060\$26.00/0

Accessible online at:

www.karger.com/nen
Gregory Kaltsas

Department of Pathophysiology

University of Athens, Mikras Asias 75

GR-11527 Athens (Greece)

Tel. +30 210746 2513, Fax +30 210746 2664, E-Mail gkaltsas@endo.gr 
Fig. 1. Pathogenesis of GC-induced bone loss.

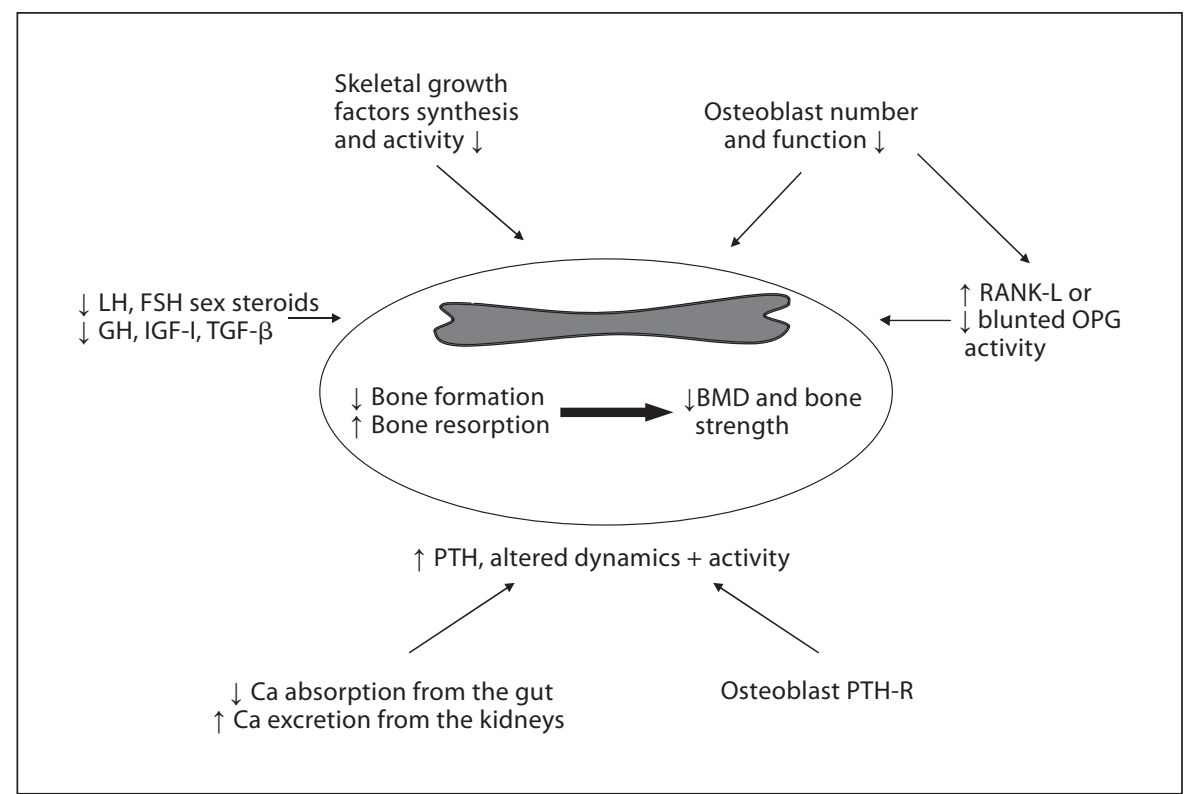

\section{Osteoporosis}

\section{Pathogenesis (fig. 1)}

The multifactorial pathogenesis of bone loss in CS depends on direct and indirect effects of GCs on bone that are not restricted to bone mineral density (BMD) alone, but also involve bone architecture, geometry and the rate of bone remodeling units (BRU), important components that together determine bone strength [1].

Sustained and prolonged hypercortisolism leads to a rapid early phase of BMD decline due to excessive bone resorption, which is followed later on by a slower and more progressive phase of impaired bone formation [2]. Besides their direct effect on BRU, GCs also reduce calcium absorption from the gastrointestinal tract due to opposition of vitamin D action and inhibition of renal tubular calcium reabsorption [2]. GCs also influence the production and action of other hormones that regulate bone and calcium metabolism such as gonadotropins, growth hormone $(\mathrm{GH})$ and insulin-like growth factor-I (IGF-I) $[2,3]$. In patients with CS, a significant reduction of lumbar spine bone density develops before involvement of the peripheral skeleton [4] because of a rapid loss of the trabecular bone [5]. The catabolic effects of GCs on muscle also contributes to fracture risk due to an increased incidence of falls secondary to muscle weakness, and loss of the stimulatory effect of muscular contraction on bone formation [2].

Skeletal Involvement in Endogenous Hypercortisolism

\section{GC Effects on Bone at the Cellular Level}

The initial event of GC effect on bone is stimulation of osteoblastic cells to increase the production of RANKL (receptor activator of NF-kappa B-RANK-ligand, which enhances osteoclastogenesis) and reduce the production of osteoprotegerin, the natural antagonist of RANKL [1]. Furthermore, GCs may act directly or through the increased expression of macrophage-colony-stimulating factor on the osteoclasts, prolonging their life span, explaining the early rapid phase of bone loss [1]. However, the most detrimental effect of GCs on bone metabolism is a profound reduction in osteoblast number and function, leading to suppression of bone formation and subsequent reduction of the amount of bone replaced in each remodeling cycle [1]. GC excess impairs osteoblastic cell differentiation by inhibiting Wnt- $\beta$-catenin signaling, and inducing the overexpression of Notch receptors thereby enhancing apoptosis of osteoblasts and osteocytes by activating caspase 3 [6]. Osteocyte apoptosis may be an additional important mechanism in GC induced fractures while the disruption of the osteocyte-canalicular network may result in a detection failure of signals that normally stimulate the replacement of damaged bone [7]. Thus, a substantial effect of GCs on osteocytes might account for a disproportionate loss of bone strength in relation to bone mass. Recent evidence has also implicated the GC-induced leukine zipper gene transcriptional factor as a crucial factor by regulating osteoblast maturation and bone turn-over [8].

Neuroendocrinology 2010;92(suppl 1):60-64 


\section{Risk of Fractures}

Approximately $30-67 \%$ of patients with CS develop fractures, mainly at the vertebrae, leading to back pain, kyphosis and loss of height [9]. The number of collapsed vertebral bodies is correlated to age at disease onset, disease duration, and urinary free cortisol levels at diagnosis even in patients with long-term cured CS [9]. In a significant number of patients, fractures may be completely asymptomatic and even develop in those with normal BMD values [10]. Men appear to be more susceptible than women, with the crucial period of developing a fracture being 2 years prior diagnosis [11]. Although it was initially suggested that the risk was higher in patients with adrenal CS this finding has not been replicated by subsequent studies [1].

Besides clinically obvious CS, a significant number of patients are increasingly being identified with alterations of cortisol secretory dynamics without exhibiting the classical signs or symptoms of CS. These patients constitute the so-called subclinical CS (SCS), mainly due to functioning adrenal adenomas, with an estimated prevalence higher than that of clinically obvious CS $(0.8 / 1,000$ vs. 1/500,000) [1]. Although not replicated consistently, several studies have documented that patients with SCS have reduced $\mathrm{BMD}$ and that this is more prominent in postmenopausal women $[1,12]$.

\section{Individual Susceptibility to Osteoporosis}

Not all individuals exposed to endogenous hypercortisolism develop osteoporosis and fractures [2]. Between the speculated mechanisms subjects carrying the N363S GC receptor polymorphism are thought to exhibit a higher sensitivity to GCs [13]. Alternatively, the activity of $11 \beta$-hydroxysteroid dehydrogenase type 1 enzyme, which acts to convert inactive cortisone to cortisol and is expressed in osteoblasts, is enhanced by GCs and leads to osteoblastic dysfunction [14].

\section{Biochemical Markers of Bone Turnover}

The net effect of bone apposition markers in patients with CS reveals a picture of reduced synthesis of new bone. Markers of bone formation, particularly osteocalcin, are substantially reduced, correlate with disease activity and increase with restoration of eucortisolism. Other markers of bone formation have shown greater variability [15], while bone resorption markers exhibit substantial variation and thus are not used routinely [1].

\section{Bone Mass}

In CS there is uncoupling between bone apposition (reduced) and resorption (normal/increased) leading to severe bone loss, especially in trabecular sites, with an estimated risk of osteoporosis of $60-70 \%$ [9]. Measurement of BMD by DEXA, particularly at the lumbar spine, presents the initial method of assessment. Vertebral CT scan can also be used to assess osteoporosis in these patients [5].

\section{Bone Architecture}

Fracture risk among prior GC users can be partly explained by BMD and the thresholds of BMD for vertebral fractures are higher in patients treated with oral GC, suggesting that other factors besides BMD may also operate [16]. Indeed, bone strength is also determined by instinct properties of bone tissue such as microdamage accumulation, bone matrix proteins and degree of mineralization, and bone turn over effects. These features are currently best accessed by peripheral quantitative computed tomography which can distinguish cortical from trabecular bone, measure the geometric properties of long bones, and estimate bone strength by calculating polar strength strain index [16]. When this method was recently applied in 10 women with CS, it revealed that all these indices were lower in patients with CS compared to controls but only in premenopausal women, suggesting a strength loss mechanism and explaining the presence of fractures even in patients with normal BMD [16]. Since the ultrasound velocity is influenced both by the organic and nonorganic bone compounds, quantitative ultrasound could be a useful tool for assessing the multifactorial bone involvement in CS; however, longitudinal studies are lacking [5].

\section{Risk Factor Stratification}

The number of collapsed vertebrae was reported to directly relate to age at disease onset, disease duration and severity based on urinary free cortisol levels, even in cured patients [5]. These parameters along with lumbar BMD measurements and possible means of assessing bone quality, and the cortisol-to-DHEAS ratio should be taken into consideration to identify patients at high risk and offer appropriate intervention [17]. Assessment of muscular strength is also highly important as muscular weakness is associated with increased fracture risk.

\section{Therapy}

The ultimate target is to prevent fractures. Appropriate interventions include calcium and vitamin $\mathrm{D}$ intake of $1,500 \mathrm{mg}$ and $800 \mathrm{IU}$ daily, respectively. Urinary cal- 
cium excretion has to be considered to avoid the development of nephrolithiasis. Administration of sex steroids has a place in the management of hypogonadal patients receiving GCs and particularly in those with SCS $[1,2]$. Similarly, GH replacement may exert a beneficial effect on bone turnover in significant number of patients with $\mathrm{CS}$ and GH deficiency [5]. However, this particular treatment has not been extensively used. Due to the increased fracture risk, a proactive preventing approach including lifestyle changes, such as tobacco cessation and reduction in alcohol consumption, exercise program, and restriction of sodium intake in the presence of hypercalciuria should be undertaken [2]. The cure of CS is reported to restore normally coupled bone metabolism and remarkably improve BMD [1]. However, normalization may take several years and may not always be complete although the fracture risk is substantially reduced over the first years following treatment [1]. In order to shorten the time of BMD recovery, particularly in high-risk patients, the use of aledronate and clodronate exhibit a higher BMD increase compared to untreated patients, suggesting that these drugs may induce a more rapid improvement in bone density than cortisol normalization alone [1]. As patients with GC induced osteoporosis have a higher risk for fractures, it has been recommended that treatment with bisphosphonates should be started with T-scores lower than -1 SD [2]. Estrogens are a reasonable approach in hypogonadal premenopausal women and in combination with bisphosphonates have additive effects on osteocyte survival, acting synergistically on the same transduction pathway although at different levels [2].

Due to the substantial reduction of bone formation, treatment with an anabolic agent that promotes bone formation and reduces vertebral and non-vertebral fractures, such as PTH, has also been suggested [2]. Although the use of teriparatide has not been yet explored in endogenous CS it has been repeatedly shown to be beneficial in patients with exogenous GC-induced osteoporosis [2]. Another potential therapeutic target is the RANKRANKL-OPG system particularly after the development of a human monoclonal antibody to RANKL (denosumab) that has been shown to reduce all osteoporotic fractures in postmenopausal patients [18].

\section{Arthropathies (table 1)}

Arthopathies related to CS are relatively rare and usually accompany osteoporosis. Abundant pseudocallus formation is frequently seen around stress fractures in
Table 1. Skeletal manifestation of Cushing's syndrome

Osteoporosis, predominantly of trabecular bones

Spontaneous symptomless fractures of ribs, pubic and ischial rami, foot bones and vertebrae

Healing of fracture with abundant pseudocallus formation

Osteonecrosis (avascular necrosis) of head of femur and humerus, distal femur and vertebrae

Suppression of growth velocity in children

the ribs, pelvis and end plates of collapsed vertebrae and is considered the hallmark of GC-induced osteoporosis [19]. Microscopically, there is a reduction in osteoblastic activity and production of a cartilaginous callus that becomes highly mineralized in an amorphous fashion [20]. Such fractures can also develop in the feet, pubic and ischial rami and uncommonly in long bones resembling pseudofractures of osteomalacia [19]. Osteonecrosis of the femoral head is most commonly encountered following exogenous GC administration although cases following endogenous hypercortisolism are increasingly being recognized; occasionally both femurs and several other joints may be involved $[1,19]$. Growth failure, weight gain and pubertal arrest are the hallmarks of CS in children and growing adolescents leading to reduced final adult height [5]. Children with hypercortisolism also develop a reduction in peak bone mass, which presents a major determinant of long-term risk of osteoporosis [5].

\section{Conclusion}

Endogenous hypercortisolism exerts a detrimental effect on bone and substantially increases the risk for spinal fractures secondary to osteoporosis. High-risk patients need to be identified and offered appropriate treatment at an early stage.

\section{Disclosure Statement}

The authors have nothing to declare. 


\section{References}

$\checkmark 1$ Chiodini I, Torlontano M, Carnevale V, Trischitta V, Scillitani A: Skeletal involvement in adult patients with endogenous hypercortisolism. J Endocrinol Invest 2008;31: 267-276.

-2 Mazziotti G, Angeli A, Bilezikian JP, Canalis E, Giustina A: Glucocorticoid-induced osteoporosis: an update. Trends Endocrinol Metab 2006;17:144-149.

$\checkmark 3$ Manelli F, Giustina A: Glucocorticoidinduced osteoporosis. Trends Endocrinol Metab 2000;11:79-85.

$\checkmark 4$ Karavitaki N, Ioannidis G, Giannakopoulos F, Mavrokefalos P, Thalassinos N: Evaluation of bone mineral density of the peripheral skeleton in pre- and postmenopausal women with newly diagnosed endogenous Cushing's syndrome. Clin Endocrinol (Oxf) 2004;60:264-270.

5 Mancini T, Doga M, Mazziotti G, Giustina A: Cushing's syndrome and bone. Pituitary 2004;7:249-252.

6 Canalis E: Mechanisms of glucocorticoid action in bone. Curr Osteoporos Rep 2005;3: 98-102.

7 Manolagas SC: Corticosteroids and fractures: a close encounter of the third cell kind. J Bone Miner Res 2000;15:1001-1005.

$\checkmark 8$ Lekva T, Bollerslev J, Kristo C, Olstad OK, Ueland T, Jemtland R: The glucocorticoidinduced leucine zipper gene (GILZ) expression decreases after successful treatment of patients with endogenous Cushing's syndrome and may play a role in glucocorticoidinduced osteoporosis. J Clin Endocrinol Metab 2010;95:246-255.
9 Shaker JL, Lukert BP: Osteoporosis associated with excess glucocorticoids. Endocrino Metab Clin North Am 2005;34:341-343ix.

10 Tauchmanovà L, Pivonello R, Di SC, Rossi R, De Martino MC, Camera L, Klain M, Salvatore M, Lombardi G, Colao A: Bone demineralization and vertebral fractures in endogenous cortisol excess: role of disease etiology and gonadal status. J Clin Endocrinol Metab 2006;91:1779-1784.

11 Vestergaard P, Lindholm J, Jorgensen JO, Hagen C, Hoeck HC, Laurberg P, Rejnmark L, Brixen K, Kristensen LO, Feldt-Rasmussen U, Mosekilde L: Increased risk of osteoporotic fractures in patients with Cushing's syndrome. Eur J Endocrinol 2002;146:51-56.

12 Torlontano M, Chiodini I, Pileri M, Guglielmi G, Cammisa M, Modoni S, Carnevale V, Trischitta V, Scillitani A: Altered bone mass and turnover in female patients with adrenal incidentaloma: the effect of subclinical hypercortisolism. J Clin Endocrinol Metab 1999;84:2381-2385.

13 Huizenga NA, Koper JW, De Lange P, Pols HA, Stolk RP, Burger H, Grobbee DE, Brinkmann AO, De Jong FH, Lamberts SW: A polymorphism in the glucocorticoid receptor gene may be associated with and increased sensitivity to glucocorticoids in vivo. J Clin Endocrinol Metab 1998;83:144-151.

14 Tomlinson JW, Walker EA, Bujalska IJ, Draper N, Lavery GG, Cooper MS, Hewison M, Stewart PM: 11beta-hydroxysteroid dehydrogenase type 1: a tissue-specific regulator of glucocorticoid response. Endocr Rev 2004;25:831-866.
15 Osella G, Terzolo M, Reimondo G, Piovesan A, Pia A, Termine A, Paccotti P, Angeli A: Serum markers of bone and collagen turnover in patients with Cushing's syndrome and in subjects with adrenal incidentalomas. J Clin Endocrinol Metab 1997;82:3303-3307.

16 Kaji H, Yamauchi M, Chihara K, Sugimoto T: Glucocorticoid excess affects cortical bone geometry in premenopausal, but not postmenopausal, women. Calcif Tissue Int 2008;82:182-190.

17 Tauchmanovà L, Pivonello R, De Martino MC, Rusciano A, De LM, Ruosi C, Mainolfi C, Lombardi G, Salvatore M, Colao A: Effects of sex steroids on bone in women with subclinical or overt endogenous hypercortisolism. Eur J Endocrinol 2007;157:359-366.

$>18$ Cummings SR, San MJ, McClung MR, Siris ES, Eastell R, Reid IR, Delmas P, Zoog HB, Austin M, Wang A, Kutilek S, Adami S, Zanchetta J, Libanati C, Siddhanti S, Christiansen C: Denosumab for prevention of fractures in postmenopausal women with osteoporosis. N Engl J Med 2009;361:756765.

19 Adachi JD, Papaioannou A: CorticosteroidInduced osteoporosis: detection and management. Drug Saf 2001;24:607-624.

20 Adachi JD: Corticosteroid-induced osteoporosis. Am J Med Sci 1997;313:41-49. 